\title{
Microbial functional traits are sensitive indicators of mild disturbance by lamb grazing
}

\author{
Xingyu Ma ${ }^{1}$ - Qiuting Zhang ${ }^{1} \cdot$ Mengmei Zheng ${ }^{2}$ - Ying Gao ${ }^{3} \cdot$ Tong Yuan $^{4} \cdot$ Lauren Hale $\mathbb{C}^{5,6}$ • \\ Joy D. Van Nostrand $\mathbb{D}^{5} \cdot$ Jizhong Zhou ${ }^{1,5,7} \cdot$ Shiqiang Wan $^{8} \cdot$ Yunfeng Yang $\mathbb{D}^{1}$
}

Received: 8 May 2018 / Revised: 19 December 2018 / Accepted: 14 January 2019 / Published online: 30 January 2019

(c) International Society for Microbial Ecology 2019

\begin{abstract}
Mild disturbances are prevalent in the environment, which may not be easily notable but could have considerable ecological consequences over prolonged periods. To evaluate this, a field study was designed to examine the effects of very lightintensity lamb grazing on grassland soil microbiomes with different soil backgrounds. No significant change $(P>0.05)$ was observed in any vegetation and soil variables. Nonetheless, hundreds of microbial functional gene families, but not bacterial taxonomy, were significantly $(P<0.05)$ shifted. The relative abundances of both taxonomic markers and functional genes related to nitrifying bacteria were also changed. The observation highlighted herein, showing a high level of sensitivity with respect to functional traits (functionally categorized taxa or genes) in differentiating mild environmental disturbance, suggests that the key level at which to address microbial responses may not be "species" (by means of rRNA taxonomy), but rather at the functional gene level.
\end{abstract}

Supplementary information The online version of this article (https:// doi.org/10.1038/s41396-019-0354-7) contains supplementary material, which is available to authorized users.

Shiqiang Wan

swan@ibcas.ac.cn

$\triangle$ Yunfeng Yang

yangyf@tsinghua.edu.cn

1 State Key Joint Laboratory of Environment Simulation and Pollution Control, School of Environment, Tsinghua University, Beijing 100084, China

2 College of Life Sciences, Henan University, Kaifeng, Henan 475004, China

3 Research Institute of Forestry, Chinese Academy of Forestry, Beijing 100091, China

4 College of Life Science, Henan Agricultural University, Zhengzhou 450002, China

5 Institute for Environmental Genomics and Department of Microbiology and Plant Biology, University of Oklahoma, Norman, OK 73019, USA

6 Agricultural Research Service, San Joaquin Valley Agricultural Sciences Center, USDA, Parlier, CA 93648-9757, USA

7 Earth Sciences Division, Lawrence Berkeley National Laboratory, Berkeley, CA 94720, USA

8 School of Life Science, Hebei University, Baoding, Hebei 071000, China
Earth's environment is continuously changing, owing to a variety of natural and anthropogenic events. A recent literature survey of 310 experimental and 68 observational investigations across a variety of habitats showed that taxonomic and functional compositions of microbiome were generally sensitive to environmental disturbances in the majority of those studies [1]. Closer examination showed that the observed shifts were usually associated with notable changes in environmental variables. However, many disturbances, e.g., introduction of low concentrations of persistent organic pollutants, are mild but may have detectable consequences in the long term [2,3]. Those important disturbances are seldom examined by scientific communities because of limited time and the challenge to quantify significant effects of mild disturbances.

To address this, we initiated a field study with very lightintensity lamb grazing in the Xilinguole grassland, a representative temperate Eurasian grassland located in Inner Mongolia, China. In the last century, the grassland has undergone severe land degradation caused by overgrazing and aeolian soil erosion [4]. To restore this grassland, the local government has restricted grazing activities since 2000 , resulting in a very light grazing intensity to the grassland. Carrying out in-depth investigation of the ecological consequences of light-intensity grazing is not only useful in addressing the fundamental scientific questions, 
but also helpful for developing proper land-use strategy of grasslands, which has broad environmental and economic impacts since livestock grazing is the predominant land-use activity in grasslands worldwide.

Here, we examine the effects of light-intensity lamb grazing under three conditions, alone or in combination with simulated aeolian soil erosion and deposition (Figure S1). We examined a total of 15 plant and soil variables and found that no variable was significantly changed by grazing $(P>0.05$, Table $\mathrm{S} 1)$.

High-throughput sequencing of PCR amplicons of the 16S rRNA gene was carried out to examine bacterial taxonomy in soil samples, resulting in 17,111 sequences per sample affiliated with 474 genera (see Supporting Information for details). Principal coordinates analysis (PCoA) showed that the overall community compositions were unchanged (Fig. 1a), which were also verified by the nonparametric dissimilarity test of adonis $(P>0.05$, Fig. 1a). Bacterial $\alpha$-diversity was similar across all of samples (Table S2). Strikingly, no OTU was significantly changed $(P>0.05)$ in relative abundance after correcting for false discovery rate $(\mathrm{FDR}<0.05)$.

A total of 31,309 genes affiliated to 676 gene families were detected by GeoChip 5.0, which contains 161,963 probes associated with a variety of microbial functional traits, such as carbon, nitrogen, phosphorus, and sulfur cycling [5]. Grazing clearly changed the overall microbial functional compositions (Fig. 1b). In addition, grazing affected microbial functional compositions through interaction with soil erosion and deposition (Table S3). These results demonstrated that shifts in community functional gene profiles were more readily detectable than shifts in microbial taxonomy in response to the mild disturbances. Opposite to previous studies showing coupling between microbial phylogenetic and functional compositions $[6,7]$, we observed no linkage between them $(r=-0.06, P=$ $0.23)$. This reinforced the recently emerging framework that microbial function and taxonomy independently vary because they are shaped by markedly different processes [8]. Cumulative evidence has revealed that similar functions may be performed in distant clades, while members of the same clade can fulfill separate metabolic functions [8, 9]. Robust as the observation is, it is still subject to the influence of technical limitations. The sensitivity of GeoChip was similar to that of quantitative PCR [10], regarded as the gold standard of nucleic acid quantification due to high specificity and sensitivity of the PCR. Therefore, GeoChip could be sensitive in detecting small differences in functional profiles. In addition, involvement of PCR amplification in $16 \mathrm{~S}$ rRNA gene sequencing can inevitably lead to technical biases.

The higher sensitivity of functional genes to light-intensity grazing might be attributed to a high dimensionality of functional profiles that leads to higher resolution, since a microbe typically has several thousands of functional genes in the genome. To test it, we examined single gene families detected by GeoChip. Out of a total of 676 gene families, $67 \%, 60 \%$, and $65 \%$ of genes showed significant changes by light-intensity grazing at control, eroded, and deposited sites, respectively, suggesting that individual functional genes were still sensitive to mild disturbance (Table S4). There was a tendency that more abundant genes were more sensitive to disturbance. Those genes that changed $(P<$ 0.05 ) by light-intensity grazing included amyA encoding $\alpha$ amylase associated with starch degradation; chitinase and acetylglucosaminidase associated with chitin degradation; cellobiase associated with cellulose degradation; $t r k G H$ and trkA encoding potassium transport system proteins; $f e o B$ encoding a ferrous iron transporter protein $\mathrm{B} ;$ nifH encoding nitrogenase reductase required for nitrogen fixation; and $p p x$ encoding exopolyphosphatase associated with polyphosphate degradation. These results suggest that microbial functional traits are more sensitive indicators of mild disturbance than 16S rRNA gene-based microbial taxonomy. Notably, only a few metabolic genes are as highly conserved as the 16S rRNA gene [11], because their functions are often less essential than the critical role of 16S rRNA gene as a protein biosynthesis machinery component. The universal presence of 16S rRNA gene in bacteria allows it as a taxonomic marker. Nonetheless, the relatively high degree of conservation in 16S rRNA gene makes it difficult to detect small variations in sequences. Alternatively, environmental conditions strongly influence functional traits but only weakly influence microbial taxonomy [8, 12], which is also observed in this study (Fig. 1). It is thus conceivable that small environmental variation caused by mild disturbance can be more faithfully reflected by changes of functional traits.

Frequent horizontal gene transfer disconnects functional genes from phylogeny [13]. Therefore, we examined amoA gene encoding the active-site subunit of ammonia monooxygenase [14], which is known to have rare horizontal gene transfer events [15]. We found that both amoA-AOA and $a m o A-A O B$ genes were altered by grazing (Fig. $1 \mathrm{c}$ and Table S4). Moreover, the amoA gene composition, from the phylum to the specie level, was significantly changed by grazing under control, eroded, and deposited conditions (the dissimilarity test of adonis, Table S5). We also examined nitrifying bacteria. A total of 23 Nitrospira, 4 Nitrobacter, and 17 Nitrosospira OTUs were identified. Nitrifying bacterial compositions were changed by grazing at the deposited site (PCoA, Fig. 1d), which were also verified by the nonparametric dissimilarity test of adonis $(P=0.01)$. Total abundance of Nitrospira increased by $34 \%$ at deposited sites, while the other two genera remained unchanged by grazing. 
Fig. 1 Principal coordinates analysis (PCoA) of a microbiome based on highthroughput 16S rRNA genesequencing data and b microbiome based on GeoChip data; $\mathbf{c}$ the nitrifying functional group based on 44 nitrifier OTUs (17 Nitrosospira, 4 Nitrobacter, and 23 Nitrospira OTUs) from 16S rRNA gene amplicon-sequencing data and d 255 detected gene probes derived from bacterial amoA gene (AOB) from GeoChip data at control, eroded, and deposited sites. The values for Axes 1 and 2 are the percentages of variations attributed to the corresponding axis. The significance labeled on figures was calculated by adonis. $P<$ 0.05 is shown in bold
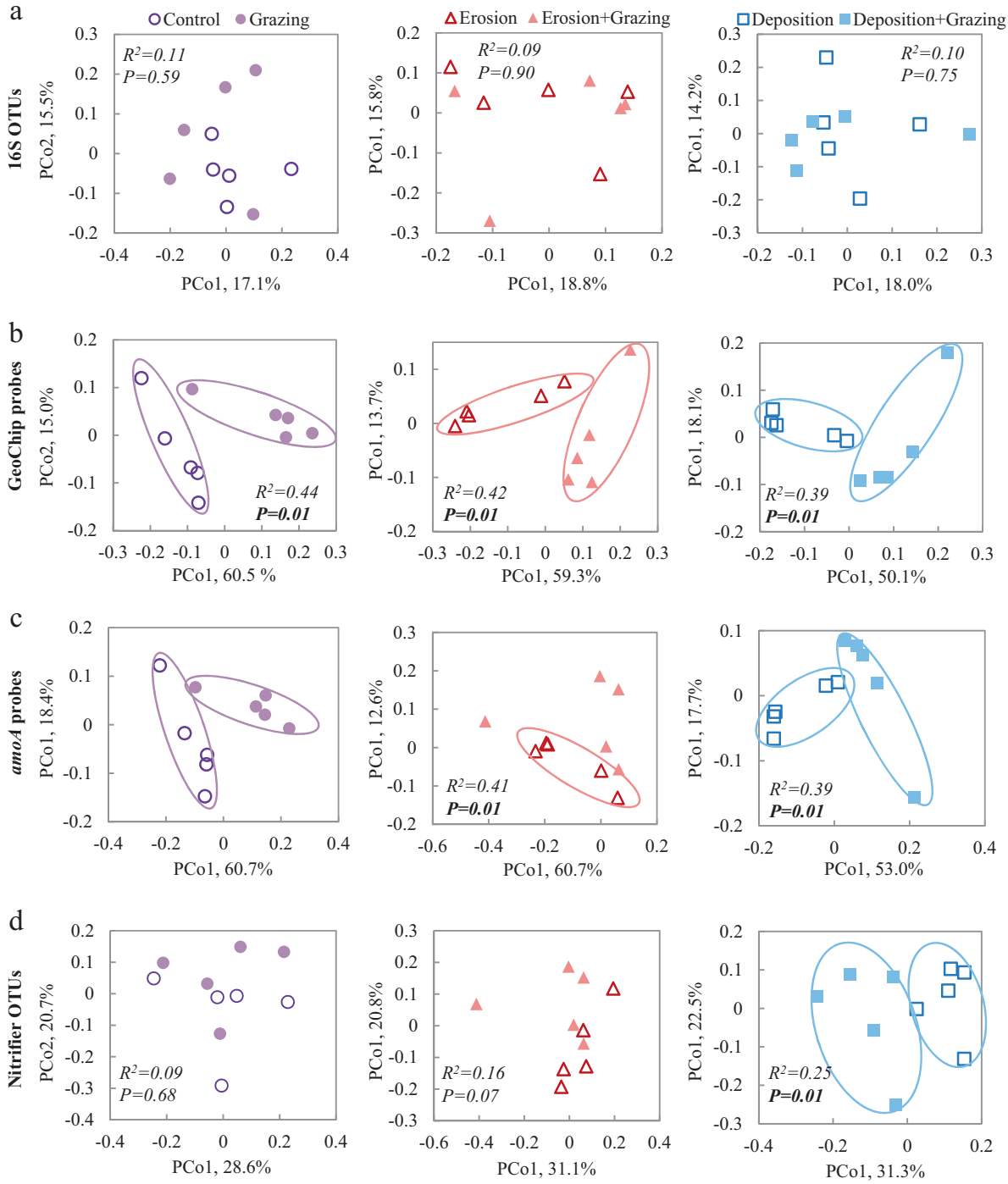

Our results highlight the importance of microbial functional response to monitor environmental disturbance. By linking the microbial functional traits with the environment, using a functional trait-centric approach to measure and explain environmental responses is becoming particularly appropriate because it is evident now that many bacterial taxa do not fit neatly into current frameworks of functional guilds [16]. Functional classification of ecological communities could increase predictability in ecological studies, as taxonomically similar communities could be functionally distinct owing to functionally divergent evolution.

\section{Data availability}

GeoChip data are available online (www.ncbi.nlm.nih.gov/ geo/) with the accession number GSE67347. MiSeq data are available in the NCBI SRA database with the accession number SRP066059.
Acknowledgements We thank two anonymous reviewers and the editor for constructive comments and suggestions to make this paper greatly improved. We also wish to thank Jack A. Gilbert for his insightful comments on the paper and staffs of Duolun Restoration Ecology Experimentation and Demonstration Station for sampling assistance. This research was supported by grants to Yunfeng Yang from the National Science Foundation of China (48125016) and the Strategic Priority Research Program of the Chinese Academy of Sciences (XDB15010102), to Shiqiang Wan from the National Science Foundation of China (31430015), and to Jizhong Zhou from the National Science Foundation of China (41877048).

Author contributions SW, JZ, and YY designed the experiments. XM, $\mathrm{LH}$, and YY wrote the paper. $\mathrm{XM}$ and $\mathrm{QZ}$ analyzed the data. MZ, YG, TY, and JVN performed the experiments. All authors have reviewed and agreed with the paper.

\section{Compliance with ethical standards}

Conflict of interest The authors declare that they have no conflict of interest. 
Publisher's note: Springer Nature remains neutral with regard to jurisdictional claims in published maps and institutional affiliations.

\section{References}

1. Shade A, Peter H, Allison SD, Baho D, Berga M, Buergmann H, et al. Fundamentals of microbial community resistance and resilience. Front Microbiol. 2012;3:417.

2. Chang H, Wan Y, Hu J. Determination and source apportionment of five classes of steroid hormones in urban rivers. Environ Sci Technol. 2009;43:7691-8.

3. Nadal M, Schuhmacher M, Domingo JL. Long-term environmental monitoring of persistent organic pollutants and metals in a chemical/petrochemical area: human health risks. Environ Pollut. 2011;159:1769-77.

4. Feng Q, Ma H, Jiang X, Wang X, Cao S. What has caused desertification in China? Sci Rep. 2015;5:15998.

5. Wu L, Yang Y, Chen S, Jason Shi Z, Zhao M, Zhu Z, et al. Microbial functional trait of rRNA operon copy numbers increases with organic levels in anaerobic digesters. ISME J. 2017;11:2874-8.

6. Fierer N, Lauber CL, Ramirez KS, Zaneveld J, Bradford MA, Knight R. Comparative metagenomic, phylogenetic and physiological analyses of soil microbial communities across nitrogen gradients. ISME J. 2012;6:1007-17.

7. Fierer N, Ladau J, Clemente JC, Leff JW, Owens SM, Pollard KS, et al. Reconstructing the microbial diversity and function of preagricultural tallgrass prairie soils in the United States. Science. 2013;342:621.
8. Louca S, Parfrey LW, Doebeli M. Decoupling function and taxonomy in the global ocean microbiome. Science. 2016;353:12727.

9. Martiny JB, Jones SE, Lennon JT, Martiny AC. Microbiomes in light of traits: a phylogenetic perspective. Science. 2015;350: aac9323.

10. Yergeau E, Kang S, He Z, Zhou J, Kowalchuk GA. Functional microarray analysis of nitrogen and carbon cycling genes across an Antarctic latitudinal transect. ISME J. 2007;1:163-79.

11. Clarridge JE. Impact of $16 \mathrm{~S}$ rRNA gene sequence analysis for identification of bacteria on clinical microbiology and infectious diseases. Clin Microbiol Rev. 2004;17:840-62.

12. Louca S, Jacques SMS, Pires APF, Leal JS, Srivastava DS, Parfrey LW, et al. High taxonomic variability despite stable functional structure across microbial communities. Nat Ecol Evol. 2016;1:0015.

13. Jain R, Rivera MC, Lake JA. Horizontal gene transfer among genomes: the complexity hypothesis. Proc Natl Acad Sci. 1999;96:3801-6.

14. Rotthauwe JH, Witzel KP, Liesack W. The ammonia monooxygenase structural gene amoA as a functional marker: molecular fine-scale analysis of natural ammonia-oxidizing populations. Appl Environ Microbiol. 1997;63:4704-12.

15. Francis CA, O'Mullan GD, Ward BB. Diversity of ammonia monooxygenase (amoA) genes across environmental gradients in Chesapeake Bay sediments. Geobiology. 2003;1:129-40.

16. Peay KG, Kennedy PG, Talbot JM. Dimensions of biodiversity in the Earth mycobiome. Nat Rev Microbiol. 2016;14:434-47. 